\title{
Glioblastoma cell line-derived spheres in serum-containing medium versus serum-free medium: A comparison of cancer stem cell properties
}

\author{
XIN HONG $^{1}$, KHALIL CHEDID ${ }^{2}$ and STEVEN N. KALKANIS ${ }^{1}$ \\ ${ }^{1}$ Department of Neurosurgery, Henry Ford Health System, Detroit, MI 48202; \\ ${ }^{2}$ School of Medicine, Wayne State University, Detroit, MI 48201, USA
}

Received May 15, 2012; Accepted July 6, 2012

DOI: 10.3892/ijo.2012.1592

\begin{abstract}
In addition to the primary culturing of cancer stem cells (CSCs) from tumor tissues, CSCs are found in established tumor cell lines. However, it is unclear how culture conditions affect CSC enrichment. Additionally, the differentiation potential of cell line-derived CSCs has not been well studied. In our study, the glioblastoma cell lines LN229, T98G, U251n and U87, were cultured as spheres in serum-containing medium (serum spheres) or serum-free medium (serum-free spheres). We found that LN229 and U251n cells expressed multiple stem cell markers such as Nestin, Sox2, Musashi-1 and CD44, and their serum spheres expressed even higher levels of Nestin, Sox 2 and Musashi-1 compared to monolayer cells and serum-free spheres. LN229 and U251n cells showed higher migration and colony formation potential compared to T98G and U87 cells, which did not express Nestin, Sox2 and Musashi-1. Serum spheres of LN229 and U251n cells also exhibited higher resistance to temozolomide compared to serum-free spheres. All tumor cell lines showed neuronal differentiation (Tuj-1 positive). Only U251n serum spheres exhibited both astrocytic (GFAP-positive) and neuronal differentiation. We conclude that sphere culture in serum-containing medium provides the most efficient enrichment of cancer stem cells. U251n cells are distinguished from other tumor cells due to their potential for multilineage differentiation.
\end{abstract}

\section{Introduction}

Increasing evidence shows that a small subset of cells within a malignant neoplasm, named cancer stem cells (CSCs) or tumorinitiating cells (TICs), are capable of initiating and driving tumor

Correspondence to: Dr Steven N. Kalkanis, Department of Neurosurgery, Henry Ford Health System, 2799 West Grand Boulevard, Detroit, MI 48202, USA

E-mail: skalkan1@hfhs.org

Key words: glioblastoma, cancer stem cells, differentiation, cell line, sphere culture growth $(1,2)$. CSCs are so defined because they possess many characteristics of normal stem cells, which have the potential for self-renewal, in vitro sphere formation, differentiation and tumorigenicity $(3,4)$. In addition they are relatively quiescent and resistant to many chemotherapy drugs, and thus become sources of tumor recurrence (5-7). CSCs are a new target of the next generation of tumor therapeutic agents.

CSCs were first isolated from leukemia (8), and then from solid tumors including glioblastoma (GBM, WHO grade IV) (9). In vitro culturing of CSCs is widely used to test their characterization and functionality. In the majority of studies CSCs are maintained and enriched in a suspension culture of spheres in serum-free medium. This sphere culture system attempts to decrease the secretion of differentiation factors and to avoid differentiation stimulation by adherence (10-12).

Although CSCs are defined by self-renewal, differentiation, and tumorigenicity, they are usually identified and isolated by stem cell markers. Markers such as Prominin-1 (CD133) and CD44 are frequently used in many types of tumors (13-15). ATP-binding cassette $(\mathrm{ABC})$ transporter (breast cancerresistance protein-1, ABCG2/BCRP1), which is a marker of side-population cells, has also been used to identify CSCs (16). Mostly, multiple stem cell markers are used to identify CSCs and these markers may differ among different tumor types. In breast cancer, for example, the tumor-initiating cells have a characteristic CD44(+)CD24(-/low) epithelial-specific antigen $($ ESA)(+) antigenic pattern (17). For prostate cancer, MDR1 and Oct-4 may be used as stem cell markers (18). In cells derived from glioblastoma, neural stem/progenitor cell markers such as nestin, Sox 2 and Musashi- 1 have been used to identify CSCs (19-22).

CSCs have been isolated not only from tumor tissues, but also from established tumor cell lines including human breast cancer (17), prostate cancer (23), epithelial ovarian carcinoma (24), melanoma (25), colon cancer $(26,27)$, brain tumor $(28,29)$ and others, and the list is expanding rapidly. Traditionally, tumor cell lines were cultured in serum-containing medium with monolayer morphology (excluding blood system tumor cells). Generally, the vigorously dividing tumor cells in serum-containing medium are considered differentiated cells. Additionally, tumor cell lines have been used in studies for decades, and cells have undergone various genomic and 
morphological changes to adapt to the in vitro growth environment. Li et al, observed several recurrent aberrations in established glioma cell lines and these aberrations are not frequently observed in primary tumors (30). Thus, cultured tumor cell lines are unlikely to contain CSCs even if they conserve some features of primary tumor biology. However, the presence of CSCs in tumor cell lines suggests that some of the cell lines may still possess stem cell potential. It is notable that the reported CSCs in tumor cell lines were often obtained from floating cells in the medium or cells which were cultured as spheres in serum-free medium. These findings have led to interesting questions. Can CSCs be re-induced from long-term cultured tumor cells? Is the sphere culture system critical for the induction of CSCs in tumor cell lines? Why do some cancer cell lines contain CSCs and others do not? Answering these questions would be helpful in understanding the development of CSCs.

In this study, we cultured the established glioblastoma cell lines as spheres in a medium with or without serum. We will examine the expression of generally accepted CSC markers in these cells and compare CSC properties such as colony formation, migration and chemotherapy resistance. Our main goal is to determine whether those CSCs identified by stem cell markers truly possess the ability to differentiate.

\section{Materials and methods}

Cell culture. LN229, T98G, U251n and U87 glioblastoma cell lines were obtained from American Type Culture Collection (ATCC). Cells were grown as monolayers when cultured in cell culture flasks with DMEM containing $10 \%(\mathrm{v} / \mathrm{v})$ fetal bovine serum (FBS), $2 \mathrm{mM}$ L-glutamine, $100 \mathrm{IU} / \mathrm{ml}$ penicillin, $100 \mu \mathrm{g} / \mathrm{ml}$ streptomycin and $1 \%$ nonessential amino acid.

To culture tumor spheres, cells were seeded in $2 \%$ poly (2-hydroxyethyl methacrylate) (poly-HEMA, Sigma)-coated cell culture flasks to prevent cell adhesion. Tumor spheres were formed either in serum-containing medium (i.e., serum sphere) or in serum-free medium (i.e., serum-free sphere). Serum spheres were cultured in the same medium as monolayer culture. Serum-free spheres were cultured in DMEM/F12 supplemented with $\mathrm{N} 2$ supplement and $0.5 \mathrm{mg} / \mathrm{ml}$ bovine serum albumin (BSA). Epidermal growth factor (EFG) and basic fibroblast growth factor (FGFb) $(20 \mathrm{ng} / \mathrm{ml}$ each) were added to the medium before culturing.

Glioma cells exhibited variant growth speeds especially when cultured as spheres. For subsequent sphere formation $100-150 \mu \mathrm{m}$ spheres were dissociated in $0.05 \%$ trypsin-EDTA and seeded at a density of 10,000 cells $/ \mathrm{ml}$. Cell culture medium was changed every 3 to 4 days. Spheres were cultured at least 2 months in serum-containing medium or serum-free medium. All cells were cultured at $37^{\circ} \mathrm{C}$ in a humidified atmosphere of $5 \% \mathrm{CO}_{2}$. Cell culture medium and additives were obtained from Invitrogen.

Cell proliferation in soft agar. To observe the clonogenic ability of cells in different culture conditions, monolayer cells and spheres were dissociated with trypsin-EDTA. A total of $1 \times 10^{4}$ cells were suspended in $0.3 \%$ low-melt agarose (Cambrex) and then seeded onto the top of $2 \%$ poly-HEMA precoated 6 -well plates (Corning). A total volume of $4 \mathrm{ml}$ of the serum-containing medium and serum-free medium were added on top of the agarose layer with cells inside. Medium was changed every 3 days. After 14 days in culture, the plates were stained by $0.05 \%$ crystal violet for colony quantification. Colonies with more than 20 cells were counted under an inverted light microscope.

Sphere migration assay. Spheroids of approximately $200 \mu \mathrm{m}$ diameter were selected for the experiments. Six to ten spheroids were used for each experimental condition in each experiment. Spheroids were transferred individually to 24-well plates containing serum or serum-free medium and allowed to migrate for $24 \mathrm{~h}$. For quantification, the mean diameter of glioma cells that had migrated from the tumor spheroid was measured in a blinded manner and expressed in relation to the mean radial distance at time $0 \mathrm{~h}$. Assays were repeated at least twice.

Western blot analysis. Glioma monolayer cells and spheres cultured in different conditions were washed two times with ice cold phosphate-buffered saline (PBS) and lysed in RIPA buffer (50 mM Tris pH 7.4, $250 \mathrm{mM} \mathrm{NaCl,} 5$ mM EDTA, 1\% NP-40, $0.1 \%$ SDS, $0.5 \%$ sodium deoxycholate, $1 \mathrm{mM}$ phenylmethylsulphonyl fluoride) containing $1 \%$ protease inhibitor cocktail (Calbiochem). Cell lysates were centrifuged at 13,000 x g for $10 \mathrm{~min}$ to remove debris and protein concentration was determined using the BCA protein assay kit (Pierce). Total protein of 10-20 $\mu \mathrm{g}$ was subjected to SDS-PAGE, transferred to polyvinylidene fluoride (PVDF) membrane, and probed with antibodies, followed by HRP-conjugated secondary antibodies. Specific proteins were detected by ECL Western Blotting Detection Reagents (GE Healthcare Biosciences). The experiments were repeated in triplicate. $\beta$-actin antibody was used as the internal protein control. Antibodies against Nestin and Sox2 were purchased from Millipore; antibodies against Musashi-1 and CD133 were the products of Cell Signaling Technology Inc.; antibodies against CD44 and $\beta$-actin were obtained from Sigma; and ABCG2 (anti-BCRP, clone BXP-21) was the product of Kamiya Biomedical Company.

For quantification of relative protein levels, X-ray film, and densitometric analysis was carried out using ImageJ software (National Institutes of Health). Each immunoblot was performed 3 to 6 times.

Cytotoxicity assay. Cytotoxicity of sphere cells responding to temozolomide (TMZ, Sigma) was assessed by release of lactate dehydrogenase (LDH) from damaged cells into the medium using a cytotoxicity detection kit (Roche Applied Science). The method was performed as specified by the manufacturer, with minor modifications. Serum spheres and serum-free spheres were dissociated with trypsin-EDTA and $2 \times 10^{5}$ single cells were seeded in $2 \%$ poly-HEMA coated 24 -well cell culture plates with the same serum concentration as their sphere culture. Cells were treated with TMZ for $48 \mathrm{~h}$ at concentrations of 800 and $1,000 \mu \mathrm{M}$. The same amount of DMSO was added into the medium as control. Then supernatants were collected and centrifuged at $10,000 \mathrm{rpm}$ for $5 \mathrm{~min}$ to remove floating cells. After sedimentation, $20 \mu \mathrm{l}$ of supernatant from samples was transferred to a 96-well, flat-bottomed plate (Costar) and mixed with $80 \mu \mathrm{l}$ PBS, to which $100 \mu 1$ of substrate mix in assay buffer was then added. Plates were kept protected from light for up to $30 \mathrm{~min}$ at room temperature. The absorbance was monitored at 


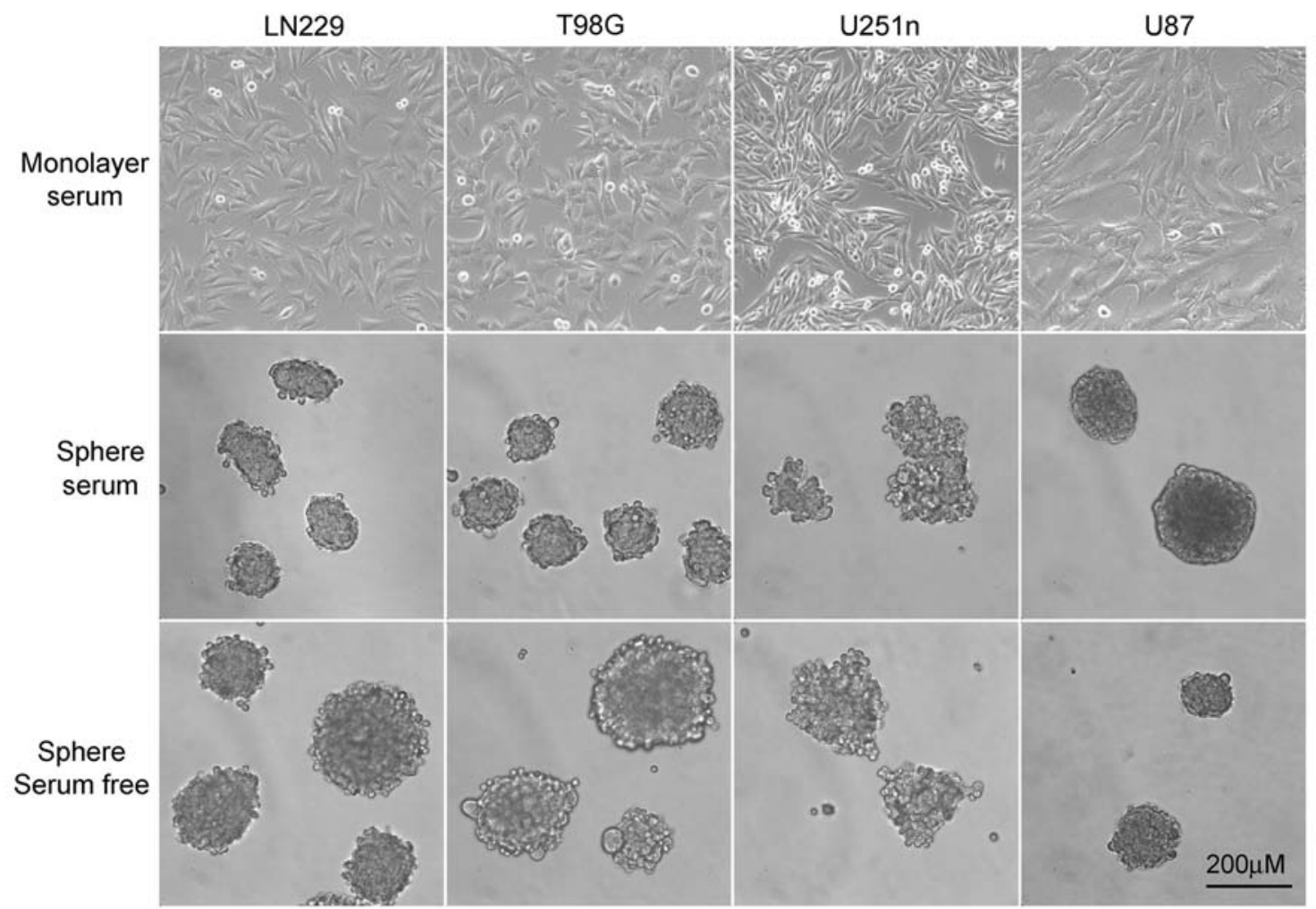

Figure 1. Cell morphology of glioblastoma cell lines in different culture conditions. Glioblastoma cells lines LN229, T98G, U251n and U87 grew as monolayers in cell culture flasks with serum-containing medium. These cells formed spheres in $2 \%$ poly (2-hydroxyethyl methacrylate)-coated cell culture flasks in serum-containing or serum-free medium.

$492 \mathrm{~nm}$ with the reference wavelength $620 \mathrm{~nm}$ in a plate reader (Multiskan MCC/340, Labsystems). The percentage of cytotoxicity was calculated relative to absorbance values for blank medium controls and values resulting from total lysis of cells by Triton X-100 (100\% cell kill) according to the following formula: Cytotoxicity $(\%)=\left(\mathrm{OD}_{\text {treatment }}-\mathrm{OD}_{\text {control }}\right) /\left(\mathrm{OD}_{\text {total }}-\mathrm{OD}_{\text {control }}\right) \times 100$.

Immunocytochemistry staining. Lab-TeK tissue culture chambers (2-well) (Nunc) were coated with poly-L-ornithine (Sigma)/fibronectin (Sigma). Serum-containing and serum-free cultured spheres were dissociated into single cells and $1 \times 10^{4}$ cells were seeded in each well. Cells were cultured with $1 \%$ FBS containing DMEM medium for 7-10 days to undergo differentiation. Then cells were fixed with $4 \%$ paraformaldehyde or methanol $(\mathrm{O} 4$ staining) and permeabilized with PBS containing $0.5 \%$ Triton $\mathrm{X}-100$ for $5 \mathrm{~min}$. Cells were stained by lineage-specific markers namely Glial Fibrillary Acidic Protein (GFAP, astrocyte marker) (Millipore), Beta III tubulin (Tuj1, neuron marker) (Covance) and $\mathrm{O} 4$ (Oligodendrocyte marker) (RnD Systems). Signals were developed with diaminobenzidine (DAB) for GFAP, Romulin AEC for Tuj1 and $\mathrm{DAB} / \mathrm{NiCl}_{2}$ for $\mathrm{O} 4$ with 4 plus HRP detection system (Biocare). Cells were counter-stained with hematoxylin (GFAP and Tuj1) and methyl-green (O4) (Sigma).

Statistical analysis. Data are expressed as the mean \pm standard deviation. Each experiment was repeated three times with duplicates. Statistical differences were evaluated using one-way ANOVA. A probability value of $\mathrm{p}<0.05$ was considered statistically significant between two groups.

\section{Results}

Nestin, Sox2 and Musashi-1 were induced in serum spheres of LN229 and U25In. Traditionally, glioblastoma cell lines were kept in $10 \%$ FBS containing medium and grown as monolayers. When cells were cultured in serum-free medium, most of the tumor cells were floating and form spheres. To make spheres of cells both in serum-containing medium and serum-free medium, we coated cell culture apparatus with $2 \%$ poly-HEMA to prevent cell adhesion. LN229, T98G and U87 formed spheres that were round with tightly connected cells. U251n formed spheres that were not exactly round from both serum-containing medium and serum-free medium. The cells in U251n spheres were loosely connected and easy to expel from the spheres. U87 cells grow extremely slowly when cultured as spheres. The number and size of U87 spheres exhibited little change during the process, even though these spheres were suspended in culture for at least 8 weeks before further testing took place (Fig. 1).

Expressions of stem cell markers were determined in cells from different culture systems by western blot assay. We found glioblastoma cells exhibited different expression patterns of stem cell markers. Nestin, Sox 2 and Mushashi-1 were expressed only by LN229 and U251n cells but not by T98G and U87 cells. Serum spheres expressed higher levels of these markers than monolayer cells and spheres from serum-free medium. Specifically, Mushasi-1 was greatly induced only in serum spheres. CD44 expressed in an opposite way to Nestin, Sox2 and Mushasi-1 in LN229 and U251n cells and serum spheres 

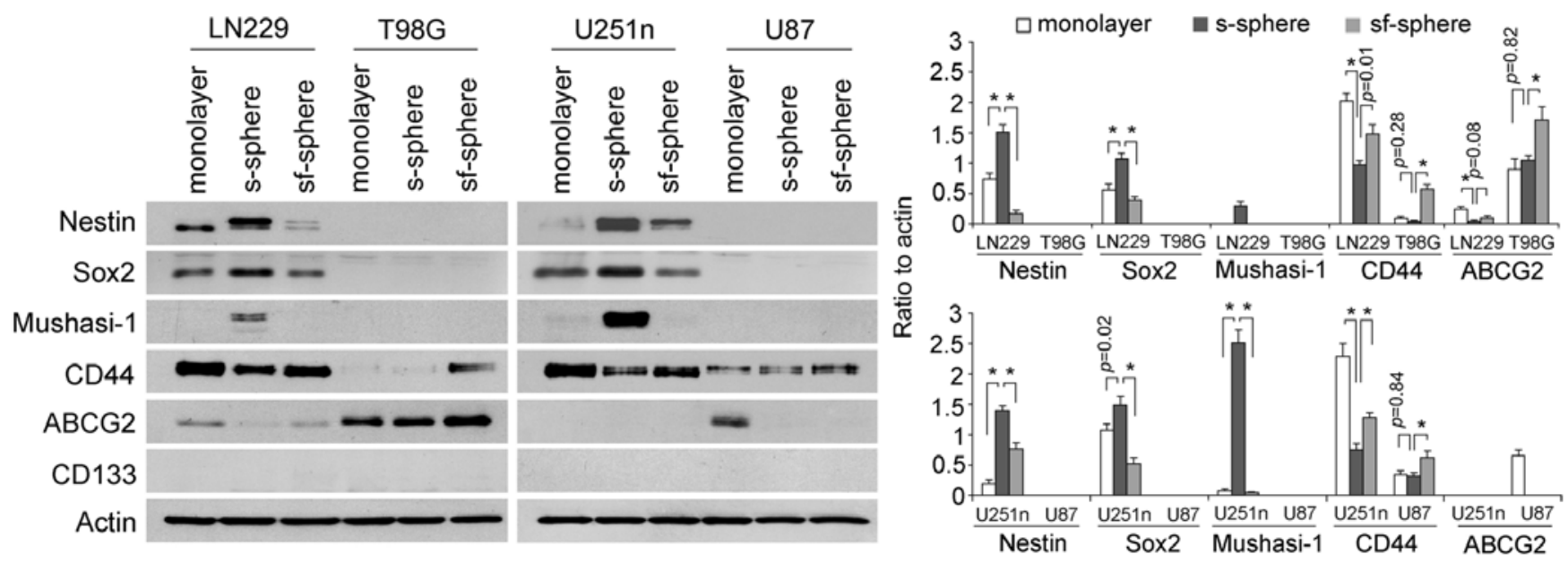

Figure 2. Western blot analysis of stem cell markers of glioblastoma cell lines cultured in different culture conditions. Protein expression levels of monolayer cells, spheres cultured in serum-containing (s-sphere) medium and serum-free (sf-sphere) medium were expressed as a ratio to actin following densitometry analysis. *Represents significant difference $(\mathrm{p}<0.01)$ between two groups.
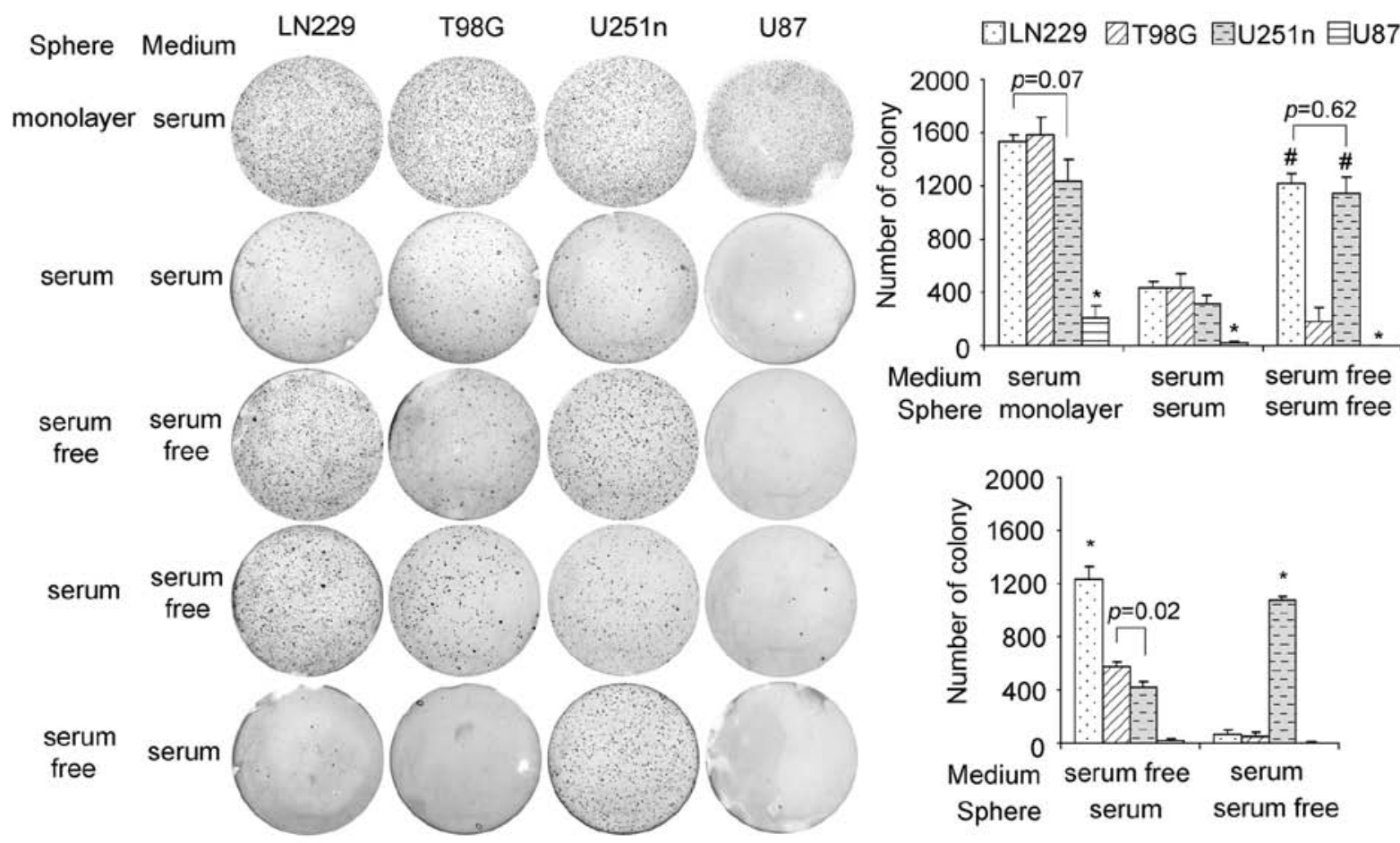

Figure 3. Colony formation of monolayer- or sphere-cultured glioblastoma cells in serum-containing or serum-free medium. Dissociated monolayer- or sphere-cells were seeded in poly (2-hydroxyethyl methacrylate)-coated 6-well plates with $0.3 \%$ soft agar in the cell culture medium. Cells were cultured in both serum-containing medium and serum-free medium. Colony numbers were counted and compared between groups. " $p<0.01$ compared with other 3 cell lines with the same culture condition (serum- or serum-free medium). ${ }^{\#} \mathrm{p}<0.01$ compared with T98G or U87 cells with serum-free medium culture.

expressed lower levels of CD44 than monolayers and serumfree spheres. CD44 was also detected by T98G and U87 cells and the highest level of CD44 was expressed by serum-free spheres. ABCG2 was detected in Ln229, T98G and U87 cells. However, ABCG2 expression was not found to be correlated with other stem cell markers. CD133 was not detected in any of the cell lines. Previously, the CD133 antibody had been successfully used in detecting primary cultured neurospheres from glioblastoma tissues (31) (Fig. 2).
Colony formation potentials in Ln229, U251n, T98G and U87 spheres. When grown as monolayers, LN229, T98G and U251n cells showed similar colony formation and U87 cells grew slower than the other three types. In the same medium as monolayer cells, the sphere cells grew more slowly than the monolayer cells but with the same growth pattern. Interestingly, serum-free sphere cells from LN229 and U251n showed a much higher rate of colonization than those from T98G and U87. LN229 and U251n sphere cells also exhibited 

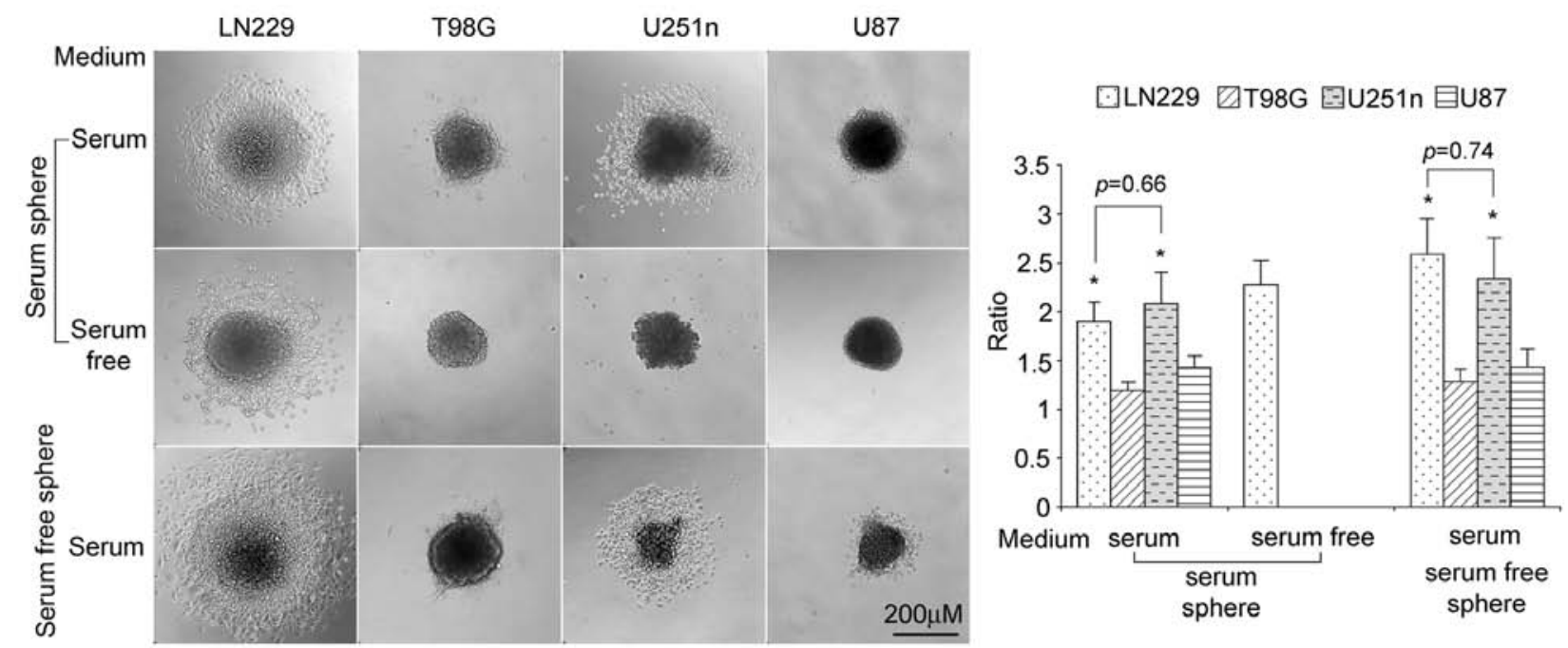

Figure 4. Cell migration of glioblastoma cell spheres in different culture media. Spheres from serum-containing and serum-free medium were placed in cell culture plates for $24 \mathrm{~h}$. Migration was expressed as the ratio of diameter of migrated cells to diameter of spheres when they were first placed. ${ }^{*}<0.01 \mathrm{compared}$ with T98G and U87 cells with the same culture medium (serum).
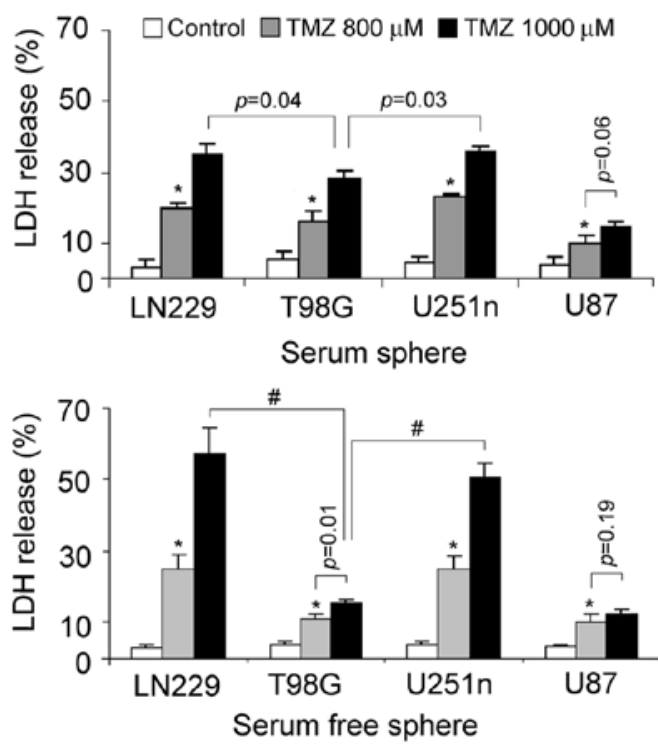

Figure 5. Cytotoxicity of glioblastoma sphere cells to temozolomide (TMZ). Spheres cultured in serum-containing medium and serum-free medium were dissociated and treated with $800,1,000 \mu \mathrm{M}$ TMZ for $48 \mathrm{~h}$. Lactate dehydrogenase (LDH) released to the cell culture medium was determined. Cytotoxicity was expressed as the percentage of released LDH to total LDH of untreated cells. ${ }^{\mathrm{p}}<0.01$ in comparison to control or $1,000 \mu \mathrm{M}$ TMZ groups unless specific $\mathrm{p}$-values were shown. "Represents significant difference $(\mathrm{p}<0.01)$ between two groups.

special colony formation potential when their growth media were changed. When changed to serum-free medium, serum spheres from LN229 presented a higher colony formation rate than the other 3 cell lines. However, when changed to serumcontaining medium, serum-free spheres of U251n exhibited a much higher rate of colonization than the other 3 cell lines, which suggests that LN229 and U251n have stronger survival ability than T98G and U87 in response to environmental changes (Fig. 3).
Migration ability of LN229, U251n, T98G and U87 spheres. We seeded spheres in plated cell culture (with no poly-HEMA coating) to test cell migration ability in serumcontaining medium or serum-free medium. When seeded in serum-containing medium, both serum spheres and serum-free spheres from LN229 and U251n cells exhibited higher rates of distant migration than those from T98G and U87. Compared with LN229 and U251 spheres, only small numbers of the T98G and U87 cells migrated outside their spheres. In serum-free medium, only serum spheres of LN229 showed migration (Fig. 4).

TMZ-induced cytotoxicity. The sensitivity of glioblastoma sphere cells to chemotherapies was evaluated by testing LDH released from damaged cells. TMZ, which is currently used in fighting glioblastoma, was chosen as the cytotoxic reagent. LN229 and U251n sphere cells from the same culture system exhibited similar cytotoxic effects following TMZ treatment. LN229 and U251n serum spheres treated after $48 \mathrm{~h}$ with $1,000 \mu \mathrm{M}$ TMZ showed cell death rates of 34 and $35 \%$, respectively. LN229 and U251n serum-free spheres treated after $48 \mathrm{~h}$ with $1,000 \mu \mathrm{M}$ TMZ showed cell death rates of 57 and $51 \%$, respectively. U87 sphere cells showed relatively lower cytotoxicity than the other 3 cells. Noticeably, serum spheres of T98G exhibited lower toxic effect than those of LN229 and U251n, while they had similar colonization rates in serum-containing medium (Fig. 5).

In vitro differentiation. Cell differentiation of sphere cells was evaluated after 7-10 days adhesive growth in medium containing $1 \%$ FBS. Both serum spheres and serum-free spheres showed neuronal differentiation. Tuj1 positive neuronal cells were observed among cell populations. Only cells from serum spheres of U251n exhibited astrocytic differentiation, which was demonstrated as GFAP-positive cells. We did not observe oligodendrocytic differentiation in any of the four cell lines in either culture system as detected by $\mathrm{O} 4$ staining (Fig. 6). 
Serum sphere LN229

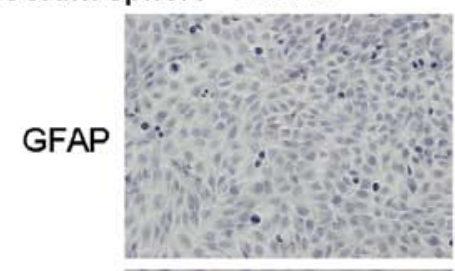

Tuj1

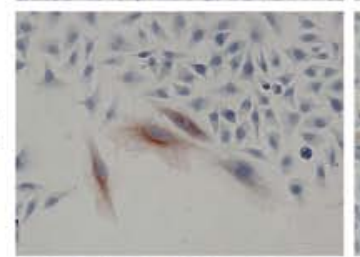

O4

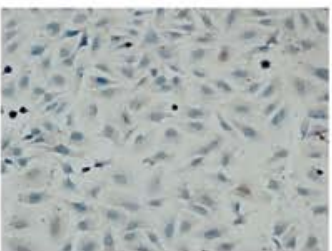

T98G
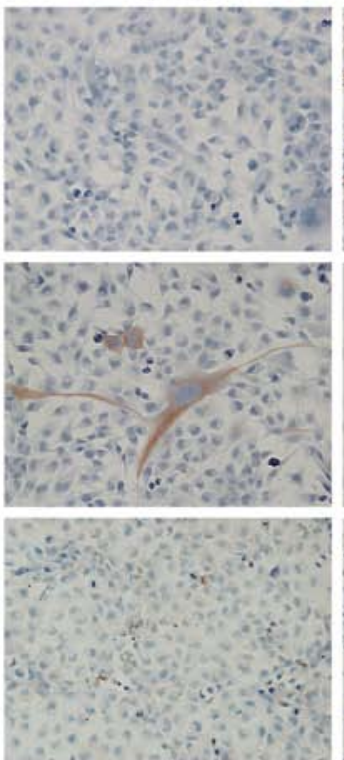

U251n
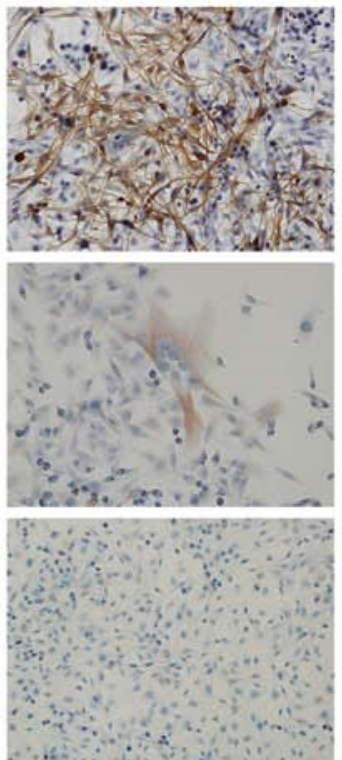
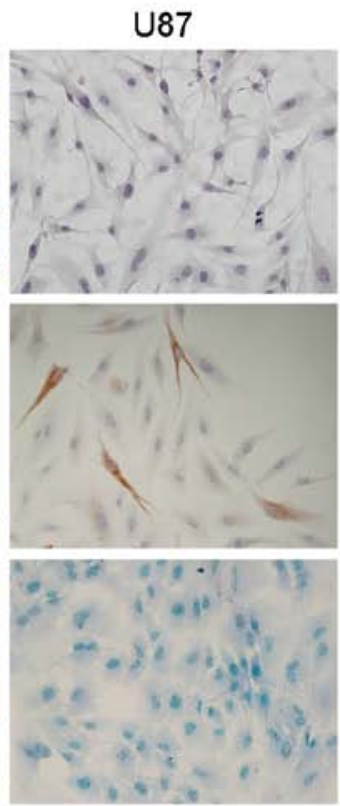

Serum-free sphere
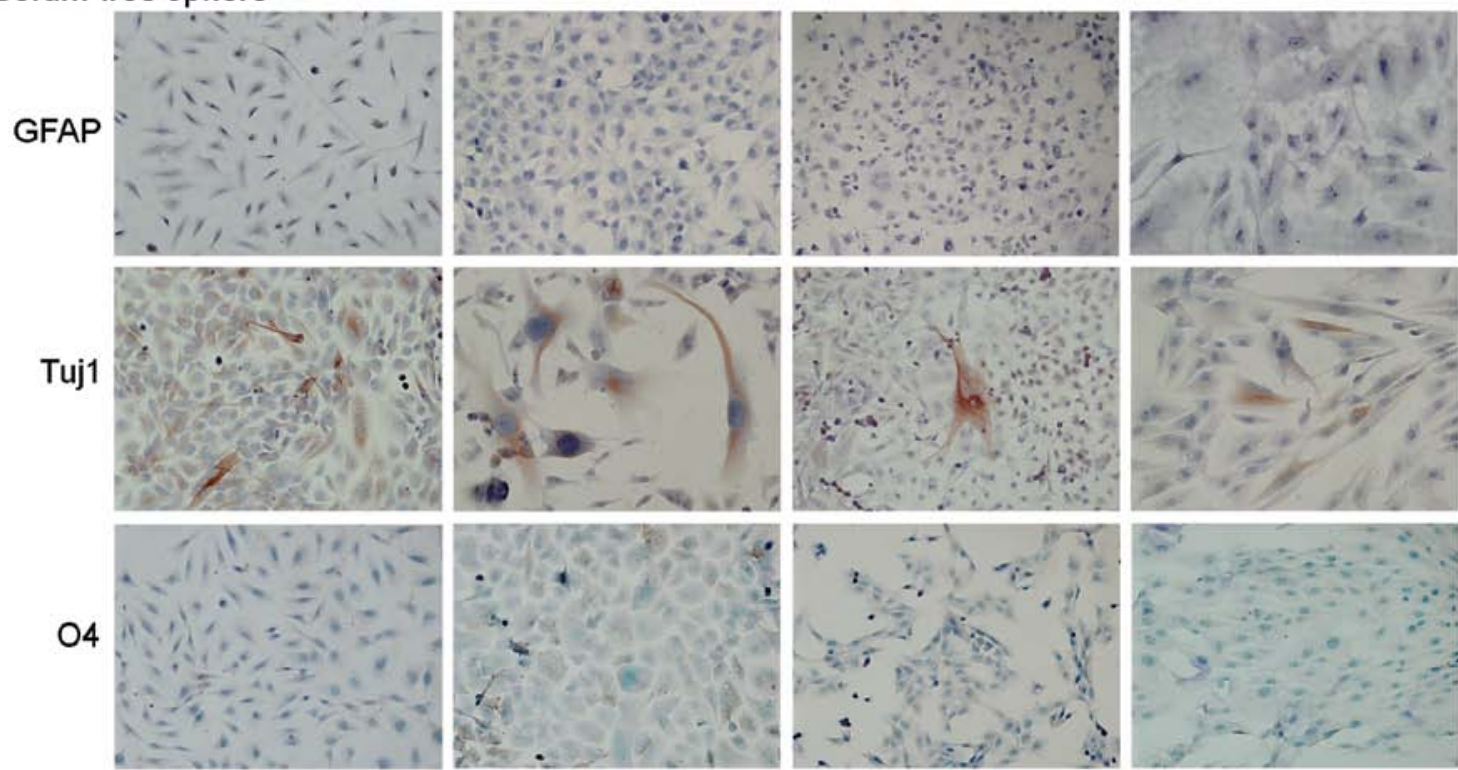

Figure 6. Differentiation of glioblastoma cell line-derived sphere cells. Spheres cultured in serum-containing medium and serum-free medium were dissociated and seeded in poly-L-ornithine/fibronectin coated slides for 7-10 days. Cells that differentiated into astrocyte, neuron and oligodendrocyte were identified using lineage-specific markers GFAP, Tuj1 and O4 by immunocytochemistry staining. Signals were developed with diaminobenzidine (DAB) for GFAP, Romulin AEC for Tuj1 and $\mathrm{DAB} / \mathrm{NiCl}_{2}$ for O4. Cells were counter-stained with hematoxylin (GFAP and Tuj1) and methyl-green (O4).

\section{Discussion}

It has been revealed that almost all tissues contain tissue-specific stem cells, which continuously generate the residential differentiated cells responsible for tissue function and homeostasis (32). The uneven cell growth and heterogeneity of tumor phenotype led to the postulation that tumors may be derived from tissue stem cells. The CSCs may proliferate to generate differentiated tumor mass and may also generate new CSCs through selfrenewal. In glioblastoma, CSCs are thought to be responsible for the invasive growth and tumor recurrence due to their resistance to chemotherapy drugs.

Sphere formation is a typical characteristic of CSCs when cultured in serum-free medium. The sphere culture of CSCs minimizes stimulation from the environment, thus keeping CSCs in an undifferentiated state. Interestingly, we found that all the tested tumor cell lines exhibited similar effects such as floating growth and sphere formation in the serum-free condition. Cell lines like U251n may not entirely float in the medium and some cells still remain adhered to the regular cell culture flasks. Additionally, we found that part of the dissociated spheres may re-attach to a non-poly-HEMA coated flask when subcultured to secondary spheres, indicating the uneven growth of the cells within the spheres.

LN229 and U251n cells are distinguished from T98G and U87 cells by expression of Nestin, Sox2, Musashi-1 and CD44. With the expression of multiple stem cell markers, LN229 and U251n cells exhibited higher migration and colonization abili- 
ties than T98G and U87 cells. Note that higher colonization was observed only in spheres cultured in serum-free medium. In serum-containing medium, the external stimulation of growth factors from serum may overwhelm the internal cell signaling. However, even with the similar expression of stem cell markers, LN229 and U251n cells showed different colonization ability when they underwent immediate medium changes. When serum spheres were placed in serum-free medium, LN229 cells showed a higher colonization rate than the other cell lines, while serum-free spheres of U251n cells placed in serum-containing medium showed significantly higher colonization than the other cell lines, indicating that LN229 and U251n cells have special abilities in adapting to environmental change. Whether the unique colonization abilities of U251n serum-free spheres in serum-containing medium predicts its multilineage differentiation potential has not been determined and needs further testing. CD133, which was used extensively as a stem cell marker, is not expressed by these cells. Recent studies indicate that both CD133-negative and CD133-positive cancer cells can initiate tumors $(33,34)$.

Serum spheres of LN229 and U251n cells exhibited more resistance to $\mathrm{TMZ}$ than their serum-free spheres. This result is consistent with our finding that serum spheres of LN229 and U251n express higher levels of stem cell markers. Cytotoxicity to TMZ is closely correlated with cell proliferation rate. Stem cell markers may be involved in the modulation of cell cycle progression and thus change cell response to chemotherapy agents. Expression levels of the drug-resistance-related gene ABCG2 is not correlated with TMZ-induced cell damage. ABCG2 also is not correlated with stem cell properties, suggesting the ABCG2 may not be crucial for stem cell function. The lower cytotoxic effects of T98G serum sphere may be due to its expression of O6-Methylguanine-DNA Methyltransferase (MGMT), which is a response to repair TMZ-induced DNA damage (35).

When checking differentiation abilities, we found that most glioblastoma cells are maintained in an undifferentiated state. Through differentiation stimulation, few tumor cells differentiated to neuronal cells. Only cells from serum spheres of U251n exhibited both astrocytic and neuronal differentiation. The limited neuronal differentiation of glioblastoma cell lines predicts that these tumor cells may originally be derived from adult neural stem or progenitor cells (36).

It is unclear why serum spheres are more stem-like than serum-free spheres. Cells cultured as spheres may change cellto-cell interaction compared to monolayer cells. In spheres, cells in the inner part of the spheres may receive less nutrients compared to the outer layer. The sphere structure formed in serum-containing medium may be more suitable for inner layer cells to transform to quiescent or dormant cells which are more stem-like. Considering the similar levels of stem cell markers expressed by LN229 and U251n, differentiation ability cannot be predicted only by the presence of stem cell markers. We assume that some cancer cells such as U251n cells have the potential to dedifferentiate and this ability is decided by the intrinsic changes within cells and may not depend only on the expression of stem cell markers. Astrocytes from p53-deficient mice were reported to dedifferentiate to stem-like cells $(36,37)$. It is possible that $\mathrm{U} 251 \mathrm{n}$ cells acquired dedifferentiation ability through genomic changes.
Our study provides evidence that some cancer cell lines may retain stem cell properties and their multi-potential ability can be restored if they are kept in the proper conditions. Serum-containing medium is a favorable environment for stem cell enrichment. Stem cell markers may predict some stem cell potential but not the ability to differentiate. U251n cells possess the most stem cell properties compared with other glioblastoma cell lines. The tumorigenic ability and invasive behavior of U251n serum spheres in vivo needs to be tested in animal models. Future studies are necessary to determine the differences between cells located at the core and the outer surface of the spheres. Understanding the molecular mechanisms of tumor dedifferentiation and formation will help provide future clinical therapies for patients with glioblastoma.

\section{Acknowledgements}

This study was supported by the Hermelin Brain Tumor Center. We thank Sue MacPhee-Gray for editorial assistance in preparing the manuscript.

\section{References}

1. Qiao B, Johnson NW, Chen X, Li R, Tao Q and Gao J: Disclosure of a stem cell phenotype in an oral squamous cell carcinoma cell line induced by BMP-4 via an epithelial-mesenchymal transition. Oncol Rep 26: 455-461, 2011.

2. Dalerba P, Cho RW and Clarke MF: Cancer stem cells: models and concepts. Annu Rev Med 58: 267-284, 2007.

3. Venere M, Fine HA, Dirks PB and Rich JN: Cancer stem cells in gliomas: identifying and understanding the apex cell in cancer's hierarchy. Glia 59: 1148-1154, 2011.

4. Reya T, Morrison SJ, Clarke MF and Weissman IL: Stem cells, cancer, and cancer stem cells. Nature 414: 105-111, 2001.

5. Marques DS, Sandrini JZ, Boyle RT, Marins LF and Trindade GS: Relationships between multidrug resistance (MDR) and stem cell markers in human chronic myeloid leukemia cell lines. Leuk Res 34: 757-762, 2010.

6. Storci G, Sansone P, Mari S, et al: TNFalpha up-regulates SLUG via the NF-kappaB/HIFlalpha axis, which imparts breast cancer cells with a stem cell-like phenotype. J Cell Physiol 225: 682-691, 2010.

7. Yip NC, Fombon IS, Liu P, et al: Disulfiram modulated ROS-MAPK and NFkappaB pathways and targeted breast cancer cells with cancer stem cell-like properties. Br J Cancer 104: 1564-1574, 2011.

8. Lapidot T, Sirard C, Vormoor J, et al: A cell initiating human acute myeloid leukaemia after transplantation into SCID mice. Nature 367: 645-648, 1994.

9. Galli R, Binda E, Orfanelli U, et al: Isolation and characterization of tumorigenic, stem-like neural precursors from human glioblastoma. Cancer Res 64: 7011-7021, 2004.

10. Singh SK, Clarke ID, Hide T and Dirks PB: Cancer stem cells in nervous system tumors. Oncogene 23: 7267-7273, 2004.

11. Leis O, Eguiara A, Lopez-Arribillaga E, et al: Sox 2 expression in breast tumours and activation in breast cancer stem cells. Oncogene 31: 1354-1365, 2012.

12. Miki J and Rhim JS: Prostate cell cultures as in vitro models for the study of normal stem cells and cancer stem cells. Prostate Cancer Prostatic Dis 11: 32-39, 2008.

13. Zhu Z, Hao X, Yan M, et al: Cancer stem/progenitor cells are highly enriched in $\mathrm{CD} 133^{+} \mathrm{CD} 44^{+}$population in hepatocellular carcinoma. Int J Cancer 126: 2067-2078, 2010.

14. Wang T, Ong CW, Shi J, et al: Sequential expression of putative stem cell markers in gastric carcinogenesis. Br J Cancer 105: 658-665, 2011.

15. Hwang-Verslues WW, Kuo WH, Chang PH, et al: Multiple lineages of human breast cancer stem/progenitor cells identified by profiling with stem cell markers. PLoS One 4: e8377, 2009.

16. Salcido CD, Larochelle A, Taylor BJ, Dunbar CE and Varticovski L: Molecular characterisation of side population cells with cancer stem cell-like characteristics in small-cell lung cancer. Br J Cancer 102: 1636-1644, 2010. 
17. Fillmore CM and Kuperwasser C: Human breast cancer cell lines contain stem-like cells that self-renew, give rise to phenotypically diverse progeny and survive chemotherapy. Breast Cancer Res 10: R25, 2008.

18. Rentala $\mathrm{S}$ and Mangamoori LN: Isolation, characterization and mobilization of prostate cancer tissue derived $\mathrm{CD} 133^{+} \mathrm{MDR}^{+}$ cells. J Stem Cells 5: 75-81, 2010.

19. Strojnik T, Rosland GV, Sakariassen PO, Kavalar R and Lah T: Neural stem cell markers, nestin and musashi proteins, in the progression of human glioma: correlation of nestin with prognosis of patient survival. Surg Neurol 68: 133-144, 2007.

20. Uchida N, Buck DW, He D, et al: Direct isolation of human central nervous system stem cells. Proc Natl Acad Sci USA 97: 14720-14725, 2000.

21. Singh SK, Hawkins C, Clarke ID, et al: Identification of human brain tumour initiating cells. Nature 432: 396-401, 2004.

22. Ying M, Wang S, Sang Y, et al: Regulation of glioblastoma stem cells by retinoic acid: role for Notch pathway inhibition. Oncogene 30: 3454-3467, 2011

23. Luk SU, Lee TK, Liu J, et al: Chemopreventive effect of PSP through targeting of prostate cancer stem cell-like population. PLoS One 6: e19804, 2011.

24. Shi MF, Jiao J, Lu WG, et al: Identification of cancer stem cell-like cells from human epithelial ovarian carcinoma cell line. Cell Mol Life Sci 67: 3915-3925, 2010.

25. Chandrasekaran S and DeLouise LA: Enriching and characterizing cancer stem cell sub-populations in the WM115 melanoma cell line. Biomaterials 32: 9316-9327, 2011.

26. Fan X, Ouyang N, Teng $\mathrm{H}$ and Yao H: Isolation and characterization of spheroid cells from the HT29 colon cancer cell line. Int J Colorectal Dis 26: 1279-1285, 2011.

27. Zou J, Yu XF, Bao ZJ and Dong J: Proteome of human colon cancer stem cells: a comparative analysis. World J Gastroenterol 17: 1276-1285, 2011
28. Zheng X, Shen G, Yang X and Liu W: Most C6 cells are cancer stem cells: evidence from clonal and population analyses. Cancer Res 67: 3691-3697, 2007.

29. Qiang L, Yang Y, Ma YJ, et al: Isolation and characterization of cancer stem like cells in human glioblastoma cell lines. Cancer Lett 279: 13-21, 2009.

30. Li A, Walling J, Kotliarov Y, et al: Genomic changes and gene expression profiles reveal that established glioma cell lines are poorly representative of primary human gliomas. Mol Cancer Res 6: 21-30, 2008.

31. deCarvalho AC, Nelson K, Lemke N, et al: Gliosarcoma stem cells undergo glial and mesenchymal differentiation in vivo. Stem Cells 28: 181-190, 2010.

32. Weissman IL, Anderson DJ and Gage F: Stem and progenitor cells: origins, phenotypes, lineage commitments, and transdifferentiations. Annu Rev Cell Dev Biol 17: 387-403, 2001.

33. Wu Y and Wu PY: CD133 as a marker for cancer stem cells: progresses and concerns. Stem Cells Dev 18: 1127-1134, 2009.

34. Clevers H: The cancer stem cell: premises, promises and challenges. Nat Med 17: 313-319, 2011.

35. Okamoto R, Takano H, Okamura $\mathrm{T}$, et al: $\mathrm{O}(6)$-methylguanine-DNA methyltransferase (MGMT) as a determinant of resistance to camptothecin derivatives. Jpn J Cancer Res 93: 93-102, 2002

36. Lee JS, Gil JE, Kim JH, et al: Brain cancer stem-like cell genesis from p53-deficient mouse astrocytes by oncogenic Ras. Biochem Biophys Res Commun 365: 496-502, 2008.

37. Moon JH, Kwon S, Jun EK, et al: Nanog-induced dedifferentiation of p53-deficient mouse astrocytes into brain cancer stem-like cells. Biochem Biophys Res Commun 412: 175-181, 2011. 